\title{
Design and Performance of a Matrix-assisted Laser Desorption/Ionization Tandem Time-of-Flight Mass Spectrometer
}

\author{
C. P. Santacruz, E. Ayala, and C. Costa-Vera \\ Departamento de Física y Astronomia, Escuela Politécnica Nacional, Ladrón de Guevara E11-253, \\ Apdo. 17-01-2759, Quito, Ecuador, Tel. 593 22567846, Fax 59322567848
}

Received on 8 June, 2006

\begin{abstract}
A matrix-assisted laser desorption/ionization tandem time-of-flight mass spectrometer has been built with the capabilities to perform structural analyzes. The first time-of-flight mass spectrometer includes a timing ion gate to select the ions to be analyzed. After the selection process, the ions are activated using an electrically floating collision cell. The fragments produced in the collision cell are analyzed in the second time-of-flight stage. A two-step mass calibration procedure is introduced that allows one to obtain accurate mass values. The characteristics of this calibration procedure make possible to automate it. The experimental results of the tandem mass spectrometer and calibration procedure using different standard peptides are presented.
\end{abstract}

Keywords: Tadem mass spectrometry; Time-of-flight; TOF/TOF; MS/MS; MALDI; Automation; Mass calibration

\section{INTRODUCTION}

At the end of the 1980's, two soft ionization techniques were introduced: electrospray ionization [1] and matrixassisted laser desorption ionization (MALDI) [2,3]. Both techniques proved to be very efficient to ionize large intact molecules as high as $10^{5} \mathrm{Da}$. The availability of such high molecular mass ions and the introduction of suitable fragmentation techniques (whereby the molecular ions can be physically divided into smaller structurally-specific fragments) expanded the application of mass spectrometry enormously. For instance, it became common to study the chemical structure of biomolecules with the aid of the so called tandem mass spectrometers. Tandem mass spectrometry $\left(\mathrm{MS}^{n}\right)$ is the use of successive multiple mass spectrometry (MS) analyses in the same or different mass spectrometers to study the molecular fragments. Thus, tandem mass spectrometry has become fundamental to the development of proteomics, genomics and metabolomics [4,5]. A tandem mass spectrometer usually consists of: (1) an ion source to generate molecular ions, (2) a device to select a desired precursor type of ion, (3) a fragmentation stage to divide the precursor ions out in specific pieces, and (4) one or more mass analyzers to determine the mass of the remaining precursor and fragment ions, in one or more successive MS steps.

There are many different types of tandem mass analyzers, but they all mix together some of the following types of mass spectrometers: linear traps [6], Fourier transform ion cyclotron resonance mass spectrometers (FTICR) [7], orbitraps [8], triple quadrupoles [9], and time-of-flight (TOF). Recently, two forms of tandem TOF mass spectrometers have emerged independently. The first of these is a hybrid quadrupole-TOF instrument, which can be used with electrospray ionization, atmospheric pressure MALDI or any other high pressure ionization methods $[10,11]$. In these instruments, the first mass spectrometer stage is a quadrupole mass analyzer which selects, accumulates, and activates the precursor ions through low energy collisions with residual gas. The resulting products are analyzed with a subsequent TOF mass analyzer. The second form of tandem TOF instruments is the dual TOF, or TOF/TOF [12-15]. In this kind of instrument, the precursor ions are selected in a first TOF $\left(\mathrm{MS}_{1}\right)$. These selected ions might then be activated through high energy collisions with neutral molecules (collision induced dissociation, CID) or by photon absorption (laser induced dissociation). Collision induced dissociation is a fragmentation technique which increases the internal energy of precursor ions by a single high energy collision (higher than $1000 \mathrm{eV}$ ) or multiple low energy collisions (around $100 \mathrm{eV}$ ) [15]. The latter case typically produces less fragmentation pathways than high energy CID [16]. Finally, the mass of the fragmentation products is determined in the second mass spectrometry stage that is usually a reflectron TOF mass analyzer $\left(\mathrm{MS}_{2}\right)$.

There are several variations of TOF/TOF instruments which are described next. The Lift TOF/TOF is a post acceleration device which lifts the potential of a region before the acceleration stage of $\mathrm{MS}_{2}$ when the precursor and fragment ions are inside it [14]. Another post acceleration TOF/TOF has a grounded fragmentation cell, and the high voltage of the acceleration region is increased from ground to high voltage [13]. In other version of TOF/TOF instruments, the precursor ions are decelerated before the collision cell, and the acceleration stage of $\mathrm{MS}_{2}$ is pulsed to high voltage [12].

In all cases concerning $\mathrm{MS}^{n}$, a strong requirement is set on having an accurate mass calibration for the parent and fragment ions, to be able of elucidating the structure [17,18]. Another very important issue is that high mass resolving power is needed to avoid ambiguities and interfering peak overlapping. Calibration in single TOF instruments is usually done by measuring the flight times of at least two ions with known masses, called standards, to determine the constant factors in the wellknown linear equation that relates the time of flight and the square root of the mass-to-charge ratio $[19,20]$. Other calibration methods use high-order polynomial [21], or quadratic corrections to reduce the mass error [22]. In tandem TOF/TOF instruments, however, it is not possible to calibrate the spectrum using the same procedure as in single TOF mass spectrometers. First, the ion selector eliminates all ions with massto-charge ratios different from that of the selected parent ion 
in the first TOF already. On the other hand, if some standards would let through the selectors, since the high voltage pulse that accelerates the precursor and fragment ions in $\mathrm{MS}_{2}$ is timed when they are in the acceleration stage of $\mathrm{MS}_{2}$, these standards will not be accelerated equally and will not have the same conditions as the precursor ion. However, peptides and proteins usually produce several known fragments, such as immonium and other low mass ions [16], which if present can be used to calibrate the CID spectra. These fragments can be identified using an external calibration procedure.

The present article describes the characteristics of a homebuilt MALDI tandem TOF/TOF mass spectrometer with a floating collision cell, reflectron and delayed extraction. In this instrument, a deceleration stage is placed before the floating collision cell to control the final collision energy of the precursor ions as they enter the cell. An external calibration procedure has been developed that uses the acceleration potentials, mass of the precursor ion, dimensions of the second TOF stage and timing of the voltages. The external calibration is used to identify immonium and low mass products, with which in turn an internal calibration procedure is performed afterwards. The report will focus in the performance of such instrument especially in the physical conditions under which the CID analysis of peptides and proteins can be made, and will highlight both the capabilities of the instrument, and some of the problems which still compromise its performance. The system has been tested using some standard commercial peptides.

\section{EXPERIMENTAL}

\section{A. Sample Preparation}

The widely used MALDI matrix alpha-cyano-4-hydroxycinnamic acid (CHCA) was purchased by Fluka and was dissolved in $50 \%$ methanol to a concentration of $10 \mathrm{~g} / \mathrm{l}$. All peptides (Sigma) were dissolved in $0.1 \%$ trifluoroacetic acid to a concentration about $10^{-5} \mathrm{M}$. Small amounts of $\mathrm{NaCl}$ were added to some samples to generate $[\mathrm{M}+\mathrm{Na}]^{+}$signals of approximately the same strength as the $[\mathrm{M}+\mathrm{H}]^{+}$signal. Samples were prepared by mixing $10 \mu l$ of matrix solution with $5 \mu l$ of analyte. Then $3 \mu l$ of the mixture was deposited on a metallic holder and left to dry at room temperature. After drying, a thin crystalline cake did form which was brought into the mass spectrometer for analysis. Mass spectra were produced by directing a focused $\mathrm{N}_{2}$-laser pulse onto the sample inside of the vacuum environment of the instrument. Over hundred spectra were added each time to improve statistics.

\section{B. Instrumentation}

The TOF/TOF instrument diagramed in Fig. 1 was constructed modifying a previously existing single TOF mass spectrometer [23]. The first TOF stage used for ion production and discrimination has a dual-stage Wiley-McLaren ion

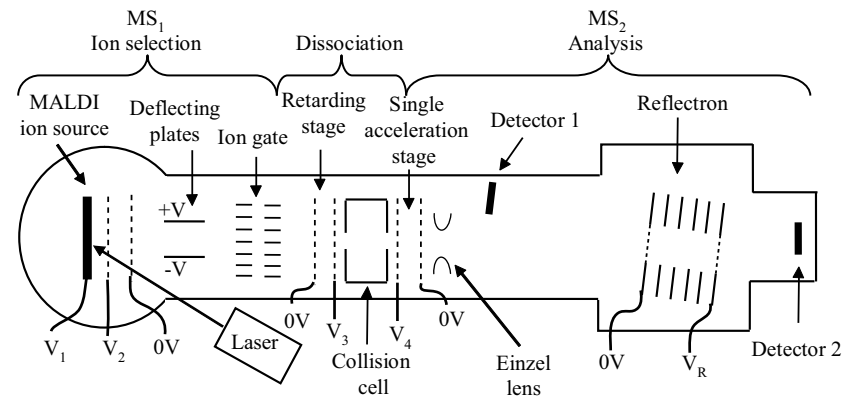

FIG. 1: A schematic representation of the home-built tandem TOF mass spectrometer. The ion source is MALDI with two consecutive acceleration stages. Timing the ion gates, the precursor ions are selected at the end of the first TOF. A floating collision cell decelerates the precursor ions to the appropriate kinetic energy and induce their fragmentation. Finally, the fragments are analyzed in the second TOF stage.

source with lengths of $7 \mathrm{~mm}$ and $13 \mathrm{~mm}$ for each stage respectively. After the ion source, a $205 \mathrm{~mm}$ long drift region, measured from the exit grid of the ion source to the center of the ion selector, separates the different ions according with their mass-to-charge ratios. Two sets of parallel deflecting plates are located after the ion source to steer the beam in two directions orthogonal to each other and to the ion path. The ion selector, which is placed at the end of the field-free drift region, operates in the manner of a dual-gate revolving door, this device has been described elsewhere [24]. Briefly, it consists of two sets of interleaved metal-ribbon-combs which are arranged in front of each other and separated by a $10 \mathrm{~mm}$ gap so that the ions can pass through. The ion selector uses the mass dependent flight time of the ions to isolate them by using timed deflectors. The sets of ribbon pieces open or close one after the other in turn in an accurately timed fashion. To open or close the gate, a transverse electric field between electrodes in the deflectors is switched off/on respectively. The first gate is normally closed and only opens when the ions of interest approach it. The second gate which comes next downstream remains open all the time and begins to close precisely at the moment when the desired ion bunch has passed through. In this way other both higher and lower mass ions are filtered out. For optimal ion selection the ion bunch should form a tightly packed group inside the gap between the gates during the switching of the fields, otherwise it can be degraded significantly if the ions are too widely spread at that position. The dual ion gate operating in revolving door configuration has a resolution around 500 that is enough to analyze mixtures of peptides.

The system uses high voltage pulses for ion acceleration, deflection, and reacceleration, which are generated by means of home-built circuits based on fast high voltage switches of the push-pull type (Behlke Electronic, Kronberg, Germany). The timing sequence of TTL pulses for the different pulsed voltages was provided by a delay generator (Model DG535, Stanford Research Systems, USA).

Fifty six millimeters after the ion selector, a $10 \mathrm{~mm}$ decel- 
eration stage is used to control the collision kinetic energy of the ions entering the collision cell. The downstream end of this stage was defined by the wall of the collision cell, which is electrically floating at the same deceleration voltage. The collision cell is cylindrical and coaxial with the ion beam and it is $80 \mathrm{~mm}$ long. Air was injected in the middle of the cell, with the aid of a pulsed gas valve (General Valve Series 9, Parker Hannifin Corporation, Fairfield, USA).

To assure enough collisions of the ions with the neutral molecules and induce their fragmentation, the valve was opened for $190 \mu \mathrm{s}$. In principle, the parent and fragment ions leave the collision cell with about the same velocity distribution before entering the acceleration region of $\mathrm{MS}_{2}$. This region performs a delayed extraction, $14 \mathrm{~mm}$ long, singleacceleration stage. The high voltage pulse for acceleration in this source increases the voltage of the electrode at the beginning of the ion source from the voltage of the collision cell up to a final higher extraction voltage.

The end electrode remains grounded for this ion source. Thus, when the ions enter the acceleration stage, there is already an electric field present generated by the deceleration voltage that is increased by activating the switch. To reduce losses and to focus axially the ion beam, an Einzel lens is placed immediately after the acceleration stage of $\mathrm{MS}_{2}$. After the ions flight through the field-free region, they enter the ion mirror. This mirror is a $289 \mathrm{~mm}$ long, tilted at $1.05 \mathrm{de}-$ grees single-stage reflectron that redirects the reflected ions into the corresponding detector. The total length of the fieldfree region of $\mathrm{MS}_{2}$ is $1183 \mathrm{~mm}$ long. Both the linear and the reflecting detectors are dual micro-channel plate arrays in Chevron configuration. The linear detector is located behind the reflectron and can be used when the reflecting field is less than the acceleration voltage or zero [23]. Ion signals were acquired with a digital oscilloscope (Tektronix TDS5000) and transferred to a computer for further analysis.

\section{Mass Calibration}

An external calibration procedure was developed considering the flight time, acceleration potentials, timing of the extraction pulse of $\mathrm{MS}_{2}$ and geometric properties of $\mathrm{MS}_{2}$. The total flight time of an ion can be expressed as:

$$
t=t_{p}+\frac{v_{4}-v_{c}}{a_{4}}+\frac{L_{2}}{v_{4}}+\frac{2 v_{4}}{a_{R}}
$$

where $t_{p}$ corresponds to the clock time at the instant when the high voltage pulse in the ion source of $\mathrm{MS}_{2}$ is triggered, $v_{c}$ is the collision velocity of the precursor ions, relative to the laboratory frame, $v_{4}$ is the velocity that ions acquire as they leave the ion source of $\mathrm{MS}_{2}, a_{4}$ is the approximately uniform acceleration of the ions in the acceleration stage of $\mathrm{MS}_{2}, L_{2}$ is the total drift length of $\mathrm{MS}_{2}$, and $a_{R}$ is the acceleration in the reflectron. The velocities $v_{c}$ and $v_{4}$ depend on the nominal acceleration voltages and distances by

and

$$
v_{c}=\sqrt{\frac{m}{M} \frac{2 z V_{c}}{m}}
$$

$$
v_{4}=\sqrt{\frac{m}{M} \frac{2 z V_{c}}{m}+\frac{2 z V_{4}}{m}}
$$

where $z V_{c}$ is the collision kinetic energy of the precursor ion, $V_{4}$ is the acceleration potential of the acceleration stage of $\mathrm{MS}_{2}, m$ is the mass of the fragment ion and $M$ is the mass of the precursor ion. It follows that Equation 1 can be expressed as

$$
t=t_{p}+\sqrt{2 \frac{m}{z}}\left(\frac{\sqrt{V_{4}+\frac{m}{M} V_{c}}-\sqrt{\frac{m}{M} V_{c}}}{\frac{V_{4}}{d_{4}}}+\frac{L_{2}}{2 \sqrt{V_{4}+\frac{m}{M} V_{c}}}+\frac{2 \sqrt{V_{4}+\frac{m}{M} V_{c}}}{\frac{V_{R}}{d_{R}}}\right)
$$

where $d_{4}$ is the length of the acceleration region of $\mathrm{MS}_{2}, d_{R}$ is the length of the reflectron and $V_{R}$ is the reflectron voltage. From this equation, the $m / z$ ratio can be obtained as

$$
\frac{m}{z}=\frac{1}{2} \frac{\left(t-t_{p}\right)^{2}}{\left(\frac{\sqrt{V_{4}+\frac{m}{M} V_{c}}-\sqrt{\frac{m}{M} V_{c}}}{\frac{V}{4}_{4}}+\frac{L_{2}}{2 \sqrt{V_{4}+\frac{m}{M} V_{c}}}+\frac{2 \sqrt{V_{4}+\frac{m}{M} V_{c}}}{\frac{V_{R}}{d_{R}}}\right)^{2}}
$$

The external calibration is an iterative procedure based on the previous equation that requires the mass of the precursor ion, its time of flight and the nominal acceleration voltages. Using Equation 5 and considering the factor $m / M=1$ for the first iteration, the mass of the fragment ions are calculated. The following iterations improve the precision of the calculated masses using Equation 5 and the values obtained in the previous iteration. After achieving high precision the iterative procedure is stopped, and the calculated masses are corrected by multiplying them for the ratio between the exact mass $\left(M_{\text {exact }}\right)$ and the calculated mass (using this procedure, $M_{\text {calculated }}$ ) of 
the precursor ions, that is

$$
\left(\frac{m}{z}\right)_{\text {corrected }}=\left(\frac{m}{z}\right)_{\text {calculated }}\left(\frac{M_{\text {exact }}}{M_{\text {calculated }}}\right)
$$

This calibration is used to identify known immonium ions or low mass fragments of amino acid residues. Once some low mass fragments are positively identified, the spectrum can be recalibrated internally using a third order polynomial equation.

$$
\left(\frac{m}{z}\right)^{1 / 2}=a_{0}+a_{1} t+a_{2} t^{2}+a_{3} t^{3}
$$

The initial values for the parameters $a_{i}$ are obtained from the external calibration procedure. Equation 5 does not require the characteristics of $\mathrm{MS}_{1}$; therefore, the external calibration procedure could be applied to any mass spectrometer working under the same conditions assumed to obtain this equation. That is, all fragment and precursor ions have the same velocity distribution when they are accelerated in the single-stage ion source of $\mathrm{MS}_{2}$. Moreover, this equation could be extended for other tandem TOF mass spectrometers, for instance, TOF with two-acceleration stages in $\mathrm{MS}_{2}$ equipped with one-stage or two-stage reflectrons.

\section{RESULTS}

The selectivity of the ion gate was tested using the protonated ion $\left([\mathrm{M}+\mathrm{H}]^{+}\right)$of substance $\mathrm{P}$ and its sodium adduct $\left([\mathrm{M}+\mathrm{Na}]^{+}\right)$. To focus the ions at the ion selector position, the extraction voltage was pulsed from $7100 \mathrm{~V}$ to $9500 \mathrm{~V}$ after a delay of $430 \mathrm{~ns}$. The ion selector was used in the revolving door configuration with two gates. The voltage of the first gate was decreased from $1000 \mathrm{~V}$ to zero with a $30 \mathrm{~ns}$ ramp, just before $[\mathrm{M}+\mathrm{H}]^{+}$entered the gate at around $6605 \mathrm{~ns}$ under our experimental conditions. The second gate was closed by increasing the voltage from zero to $1000 \mathrm{~V}$ immediately after the $[\mathrm{M}+\mathrm{H}]^{+}$ions passed through the gate at around $6635 \mathrm{~ns}$ (Fig. 2B). The 30 ns open time was enough to let only the desired precursor through with a resolving power of about 500 . By changing the timing of both gates to $6685 \mathrm{~ns}$ and 6715 ns respectively, $[\mathrm{M}+\mathrm{Na}]^{+}$was selected instead (Fig. 2C). Trying shorter selection windows for the ion gate, resulted in decreased sensitivity, and did not improve effectively the resolution, it was not possible, thus, to select just one isotopic component of the substance $\mathrm{P}$ precursor ion.

The characteristics of the instrument in tandem configuration were studied using substance P, angiotensin I, bombesin, and insulin chain B. All data presented here were acquired with $9 \mathrm{kV}$ in $\mathrm{MS}_{1}$ and $13 \mathrm{kV}$ in $\mathrm{MS}_{2}$. The operating conditions of $\mathrm{MS}_{1}$ (voltages and delays) were chosen to focus the precursor ions close to position of the ion selector at the end of $\mathrm{MS}_{1}$.

To reduce the laboratory collision energy of precursor ions to $2 \mathrm{keV}$, the collision cell was electrically floating. After collision of the precursor ions with molecules of air, the precursor and fragment ions were separated from each other in $\mathrm{MS}_{2}$. To

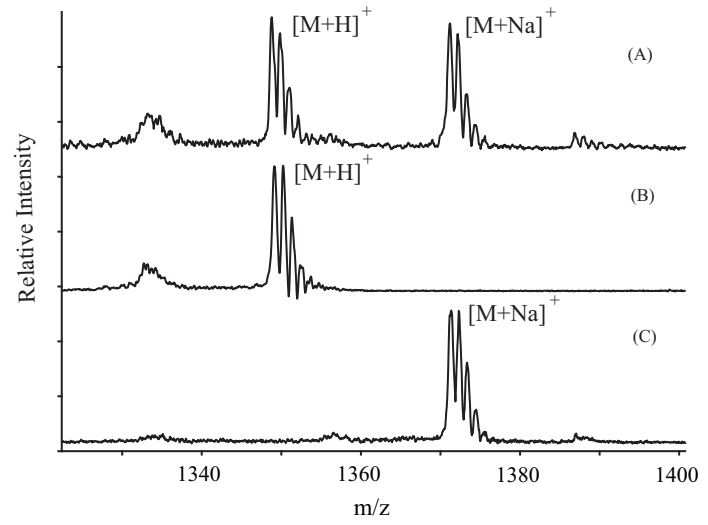

FIG. 2: Ion selector mass spectra of substance $\mathrm{P}$ showing the $\mathrm{m} / \mathrm{z}$ region spanning $[\mathrm{M}+\mathrm{H}]^{+}$, and $[\mathrm{M}+\mathrm{Na}]^{+}$. (A) Both gates off (grounded), (B) isolated $[\mathrm{M}+\mathrm{H}]^{+}, t_{\text {gate }}=6.605 \mu \mathrm{s}, V_{\text {gate }}=1 \mathrm{kV}$, and (C) isolated $[\mathrm{M}+\mathrm{Na}]^{+}, t_{\text {gate }}=6.685 \mu \mathrm{s}, V_{\text {gate }}=1 \mathrm{kV}$. Delayed extraction conditions: pulse $=9.5 \mathrm{kV}$, second acceleration stage $=7.1$ $\mathrm{kV}$, delay $=0.430 \mu \mathrm{s}$.
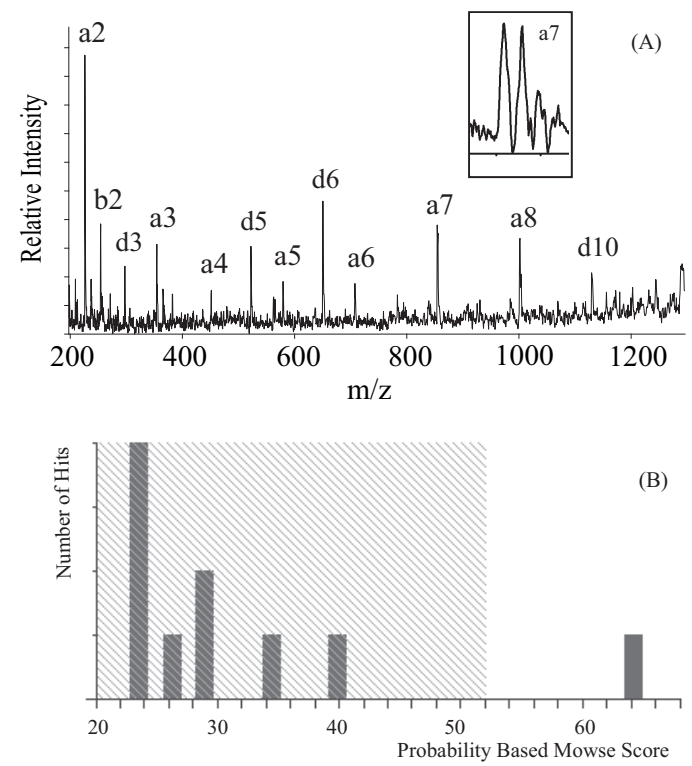

FIG. 3: (A) The CID spectrum of substance P (RPKPQQFFGLM) showing extend fragmentation. The spectrum is full of $a$ and $d$ type fragments caused by the position of the argenine residue in the $\mathrm{N}$ terminal. (B) Probability based Mowse score after database search.

accelerate the precursor and fragment ions, the timing of the voltage pulse of $\mathrm{MS}_{2}$ was set equal to the flight time of the precursor ions to the beginning of the acceleration region.

Mass spectra were calibrated externally by using the procedure described above. With this procedure some immonium or low mass fragment ions were identified and, in combination with the mass of the precursor ion, it was possible to recalibrate the spectra internally. With the final mass of the fragments, the peptide sequence was obtained by using a public access search engine (MASCOT, MS/MS Ions Search).

A high energy CID spectrum of substance $P$ is shown in Fig. 3A. In this case, the reflectron voltage was $15700 \mathrm{~V}$ to 
focus fragments with masses around $1000 \mathrm{Da}$ at the detector position. Under these conditions, the mass resolving power for ions with mass $226 \mathrm{Da}$ is 850 , for ions with mass $1001 \mathrm{Da}$ is 1900 , and for precursor ions (1348 Da) is 1450 . Using the external calibration was possible to identify some immonium ions and low mass fragments (Table I) corresponding to amino acids present in the peptide sequence. After internal calibration, the fragment list was analyzed with MASCOT. From the results list, an unnamed protein product corresponding to the substance $P$ sequence (Bos Taurus, access number gi1364200, NCBInr database) got the highest Mowse score (Fig. 3B) [25].

Figures 4A and 5A show the high energy CID spectra of angiotensin I and bombesin. The mass resolving powers range from 900 to 2000 in both cases. The analyses of the fragment list using the public access search engine for these peptides are shown in Figs. 4B and 5B respectively, and the results for the database search are summarized in Table I. The root mean square (RMS) errors, obtained using the external calibration, were between $700 \mathrm{ppm}$ to $1400 \mathrm{ppm}$. The internal calibration procedure reduced the mass error to values four to five times. Insulin chain B is a larger peptide which was not possible to identify using the above indicated procedures. In this case, the limitation was mainly the low intensity of the generated fragment ions and the missed cleavage sites. This low intensity reduced mass accuracy by introducing ambiguities in the peak centroid determination from the subjacent noise in the spectra. However, some low mass ions were identified using the external calibration procedure, and the internal calibration reduced the RMS mass error two times. Besides ion intensity, ripples in the high voltage power supplies contribute to reduce the mass accuracy and this effect is exacerbated as masses get larger. A combination of these and other technical factors (limitations in the manufacturing of the acceleration stages, alignment of the ion optics, and grid effects) seem to be limiting the mass resolving power to a value below 3000 in all our spectra. To increase the ion intensity, the ion transmission from the ion source, through the second TOF to the detector could be improved by using proper ion optics and/or gridless acceleration regions for $\mathrm{MS}_{1}$. Increasing the ion transmission, it is possible to get higher mass resolving power for tandem spectra. Besides, the overall resolving power in TOF/TOF instruments could be improved by using a two-stage acceleration region in $\mathrm{MS}_{2}$ [26].

In our case, high energy CID of substance $P$, angiotensin I, and bombesin using air as target molecules produced extended fragmentation. The mass error after the external calibration procedure let us to identify some low mass fragments and gave initial values for the coefficients of the final third order internal calibrating equation. Even with a modest resolution of around 2000, the information carried by the fragment ions was enough to identify peptides in the 800-1700 Da mass range with the aid of public access databases and search engines. The RMS error obtained using the external calibration was between $300 \mathrm{ppm}$ to $900 \mathrm{ppm}$. The internal calibration procedure reduced the mass error to values four to five times.
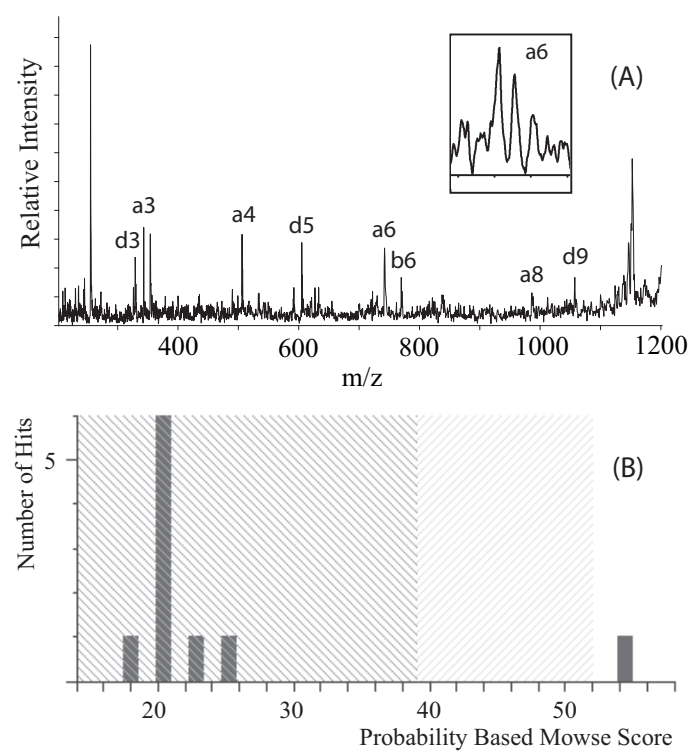

FIG. 4: (A) The CID spectrum of angiotensin I (DRVYVHPFNL). The argenine close to the $\mathrm{N}$-terminal produce $a$ and $d$ type fragments. The intensity of the fragments is low, but it was enough to produce a positive identification. (B) Probability based Mowse score after database search.
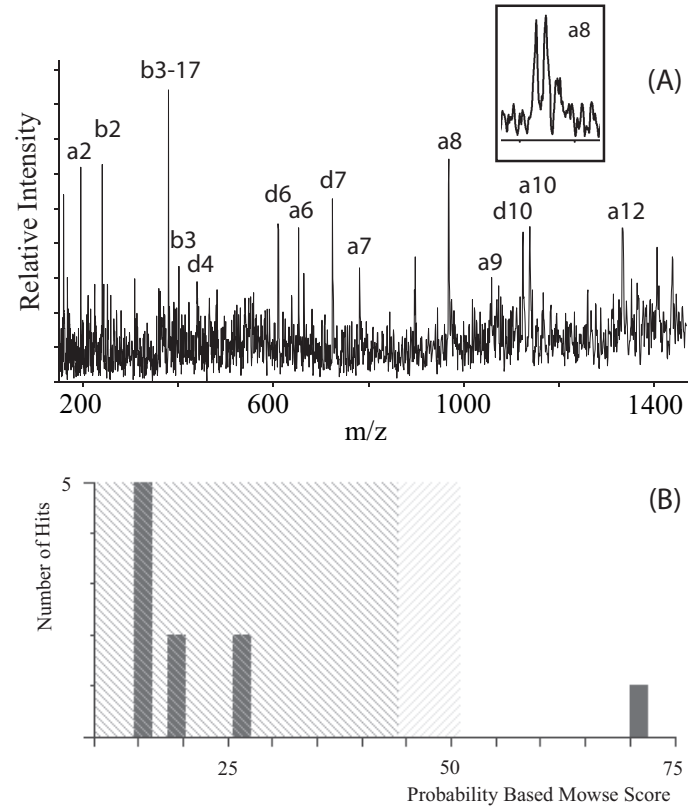

FIG. 5: (A) The CID spectrum of bombesin (pEQRLGNQWAVGHLM) shows similar characteristics to peptides containing $\mathrm{N}$-terminal argentine residue, that is, there are abundant $a$ and $d$ fragments. (B) Probability based Mowse score after database search.

\section{CONCLUSIONS}

The present article describes the characteristics of a homebuilt tandem time-of-flight mass spectrometer using a novel calibration procedure. The dual ion gate in revolving door configuration has a resolution around 500 that is enough to 


\begin{tabular}{ccccc}
\hline Peptide & Low mass Ions & $\begin{array}{c}\text { RMS mass error } \\
\text { External Cal. }\end{array}$ & $\begin{array}{c}\text { Internal Cal. } \\
\text { [ppm (Da) }\end{array}$ & $\begin{array}{c}\text { Access number } \\
\text { NCBInr database }\end{array}$ \\
\hline Substance P & $70,101,112,129$ & $1224(0.31)$ & $152(0.09)$ & gi13634200 \\
Angiotensin I & $110,129,136$ & $708(0.25)$ & $203(0.10)$ & gi2223630 \\
Bombesin & $86,110,129$ & $890(0.25)$ & $165(0.07)$ & gi229440 \\
Insulin Chain B & 70,110 & $1380(2.6)$ & $845(1.5)$ & \\
\hline
\end{tabular}

TABLE I: Summarized results showing the identified immonium ions using the external calibration, and the RMS mass error using the external and internal calibrations.

analyze mixtures of peptides. The electrically floating collision cell produces extended fragmentation for further analysis. The system has a mass resolving power of around 2000 that was enough to identify three of four peptides tried in this work with a regular Mowse score. More demanding applications with 'real life' samples will require substantially increment of the resolving power and mass accuracy to make the identification reliable and less ambiguous. For resolution improvement, it is necessary to improve the ion optics and ion detection systems of the instrument. Other improvements are required in the electronics and computer controlled data acquisition parts to increase the throughput of the system. The two-steps calibration procedure uses an external calibration to identify low mass ions, followed by an internal calibration using the information gathered in the previous step. The external calibration does not consider the characteristics of the first mass spectrometer; it uses only the nominal acceleration voltages, the mass of the precursor ion and the characteristics of the second TOF mass spectrometer. Thus, it is possible to have an automated external mass calibration for further recalibration at any operating conditions. In our case the internal calibration is a third-order polynomial, however, any other equation could be used to calibrate the mass spectra. Further developments may include an automated calibration procedure for the spectra that would combine the external calibration procedure, a peak recognition algorithm, and a reliable internal calibration.

\section{Acknowledgments}

The financial support from the International Program in the Physical Sciences of Uppsala University is gratefully acknowledged.
[1] J. B. Fenn, M. Mann, C. K. Meng, S. F. Wong, and C. M. Whitehouse, Science 246, 64 (1989).

[2] K. Tanaka, H. Waki, Y. Ido, S. Akita, Y. Yoshida, T. Yoshida, and T. Matsuo, Rapid Commun. Mass Spectrom. 2, 151 (1988).

[3] M. Karas and F. Hillenkamp, Anal. Chem. 60, 2299 (1988).

[4] J. Peng and S.P. Gygi, J. Mass Spectrom. 36, 1083 (2001).

[5] T. Werner, Mass Spectrom. Rev. 23, 25 (2004).

[6] W. Paul, Rev. Modern Physics 62, 531 (1990).

[7] S. Guan and A.G. Marshall, Int. J. Mass Spectrom. Ion Proc. 146:147, 261 (1995).

[8] A. Makarov, Anal. Chem. 72, 1156 (2000).

[9] Y. Huang, G. Li, S. Guan, and A.G. Marshall, J. Am. Soc. Mass Spectrom. 8, 962 (1997).

[10] A. Shevchenko, A. Loboda, A. Shevchenko, W. Ens, and K.G. Standing, Anal. Chem. 72, 2132 (2000).

[11] I. V. Chernushevich, A. V. Loboda, and B. A. Thomson, J. Mass. Spectrom. 36, 849 (2001).

[12] M. Vestal, P. Juhasz, W. Hines, and S. Martin, An Improved Delayed Extraction MALDI-ToF MS for PSD and CID, Proceedings of the 46th ASMS Conference on Mass Spectrometry and Allied Topics, page 35, May 32 - Jun 41998.

[13] K. F. Medzihradszky, J. M. Campbell, M. A. Baldwin, A. M. Falick, P. Juhasz, M. L. Vestal, and A. Burlingame, Anal. Chem. 72, 552 (2000).

[14] D. Suckau, A. Resemann, M. Schuerenberg, P. Hufnagel, J. Franzen and A. Holle, Anal. Bioanal. Chem. 376, 952 (2003).
[15] L. Sleno and D.A. Volmer, J. Mass Spectrom. 39, 1091 (2004).

[16] I. A. Papayannopoulos, Mass Spectrom. Rev. 14, 49 (1995).

[17] D. H. Russell and R. D. Edmondson, J. Mass Spectrom. 32, 263 (1997).

[18] K. R. Clauser, P. Baker, and A.L. Burlingame, Anal. Chem. 71, 2871 (1999).

[19] R. J. Cotter, Anal. Chem. 64, 1027A (1992).

[20] G. Montaudo, M. S. Montaudo, C. Puglisi, and F. Samperi, Anal. Chem. 72, 552 (2000).

[21] C. Costa-Vera, R. Zubarev, H. Ehring, P. Håkansson, and B.U.R. Sunqvist, Rapid Commun. Mass Spectrom. 10, 1429 (1996).

[22] C. A. Hack and W. H. Benner, Rapid Commun. Mass Spectrom. 13, 1304 (2002).

[23] C. Costa-Vera, Matrix-assisted Laser Induced Desorption/Ionization of Biomolecules, Departamento de Física - Escuela Politécnica Nacional, Departamento de Física, Ladrón de Guevara E11-253, Quito, Ecuador, 1999.

[24] C. K. G. Piyadasa, P. Håkansson, T. R. Ariyaratne, and D. F. Barofsky, Rapid Commun. Mass Spectrom. 12, 1655 (1998).

[25] D. J. C. Pappin, P. Hojrup, and A. J. Bleasby, Curr. Biol. 3, 327 (1993).

[26] C. Santacruz, E. Ayala, and C. Costa-Vera, Tandem TOF/TOF Mass Spectromter: A Mathematical Approach, Publication submitted, 2006, 